\title{
A Post hoc analysis on rhythm and high intensity interval training in cardiac resynchronization therapy
}

Xavier Melo, Ana Abreu, Vanessa Santos, Pedro Cunha, Mário Oliveira, Rita Pinto, Miguel Carmo, Bo Fernhall \& Helena Santa-Clara

To cite this article: Xavier Melo, Ana Abreu, Vanessa Santos, Pedro Cunha, Mário Oliveira, Rita Pinto, Miguel Carmo, Bo Fernhall \& Helena Santa-Clara (2019): A Post hoc analysis on rhythm and high intensity interval training in cardiac resynchronization therapy, Scandinavian Cardiovascular Journal, DOI: 10.1080/14017431.2019.1630747

To link to this article: https://doi.org/10.1080/14017431.2019.1630747

Jun 2019.

Published online: 21 Jun 2019.

Submit your article to this journal $₫$

Џ Article views: 5

View Crossmark data ¿ 


\title{
A Post hoc analysis on rhythm and high intensity interval training in cardiac resynchronization therapy
}

\author{
Xavier Melo ${ }^{a, b}$ (D), Ana Abreuc, Vanessa Santos ${ }^{a}$, Pedro Cunha $^{c}$, Mário Oliveirac, Rita Pinto ${ }^{a}$, Miguel Carmo ${ }^{c, d}$, \\ Bo Fernhall ${ }^{\mathrm{e}}$ and Helena Santa-Clara ${ }^{\mathrm{a}}$ \\ ${ }^{\text {a}}$ Faculdade de Motricidade Humana, Universidade de Lisboa, CIPER - Centro Interdisciplinar de Estudo da Performance Humana, Portugal, \\ Lisboa, Portugal; ' ${ }^{b}$ Ginásio Clube Português, GCP Lab., Lisboa, Portugal; 'Departamento de Cardiologia, Hospital Santa-Marta, Lisboa, \\ Portugal; ${ }^{d}$ Faculdade de Ciências Médicas, Universidade Nova, CEDOC - Centro de Estudos de Doenças Crónicas, Lisboa, Portugal; \\ eIntegrative Physiology Laboratory, College of Applied Health Sciences - The University of Illinois at Chicago, Chicago, IL, USA
}

\begin{abstract}
Objectives. Evaluate the effects of a 6-month High Intensity Interval Training (HIIT) program on (1) functional capacity and health-related quality of life, (2) multiple blood biomarkers, (3) echocardiographic parameters, and (4) exercise performance, in patients in cardiac resynchronization therapy (CRT) stratified by the presence of atrial fibrillation (AF), targeting the following questions: (1) Does CRT provide similar benefits in patients in AF and sinus rhythm (SR)?; and (2) Does HIIT provides similar benefits in patients in AF and SR? Design. Estimates were available at baseline and 6 months after CRT implantation in 37 patients with heart failure. Patients were randomized after CRT to a 24-week HIIT group or to a usual care group (CON). In this sub-analysis, HIIT $(A F=7 ; S R=11)$ and $C O N(A F=9 ; S R=10)$ were stratified by the presence of AF. Results. Patients in AF benefitted to a lesser degree from CRT in functional status than patients in SR (23.8-46.0\%). However, HIIT induced superior improvements in patients in AF compared to CON (23.9-61.0\%). Decreases in TNF- $\alpha$ (8.5-42.9\%), BNP (15.3-34.6\%) and left ventricular mass (9.6-26.2\%) were only observed in patients in SR, whereas increases in peak oxygen uptake were only observed in patients in AF (19.5-23.2\%). HIIT improved exercise capacity (8.8-59.4\%) in patients in SR. Conclusions. Patients in AF or SR undergoing CRT demonstrated distinct benefits from device implantation and from HIIT as an adjunctive therapeutic strategy. This suggests that both mainstay and adjunctive therapeutics may need to be managed differently in patients in AF and SR.
\end{abstract}

\section{ARTICLE HISTORY}

Received 16 January 2019

Revised 31 May 2019

Accepted 5 June 2019

\section{KEYWORDS}

Sinus rhythm; atrial fibrillation; exercise; heart failure; reduced ejection fraction; cardiac rehabilitation

\section{Introduction}

Cardiac resynchronization therapy (CRT) leads to improvements in exercise capacity, functional class, peak oxygen consumption $\left(\mathrm{VO}_{2}\right)$, hemodynamic measures, health-related quality of life [1] and mortality [2]. However, observational and intervention studies suggest that the benefits in clinical or surrogate outcomes of CRT are reduced among patients with a history of atrial fibrillation (AF) [3-5]. AF precludes atrioventricular optimization of CRT and may decrease cardiac output [6]. During AF, atrioventricular synchrony, one of the main benefits of the CRT, is lost [7]. Importantly, randomized, controlled clinical outcome trials of CRT have almost always excluded patients in AF. This is disconcerting as the prevalence of $\mathrm{AF}$ in patients with heart failure is high and these patients have reduced survival and more advanced symptoms of heart failure [8].

Exercise training leads to further improvements in functional capacity, hemodynamic measures, and health-related quality of life in addition to the improvements seen following CRT $[9,10]$. However, this is not an universal finding. Our group has shown recently that 6 months of HIIT in patients in CRT did not further improve indices of functional capacity, health-related quality of life, and left ventricular structure and function, compared to CRT alone [11]. HIIT led to further improvements in exercise performance, but it remains unclear whether patients with and without regular, organized atrial activity derive the same benefits from HIIT, as it is known that atrioventricular timing may influence the response to CRT $[3,5]$. Here, we report the results of a post hoc analysis of the previous randomized control trial [11], with a focus on whether patients in AF enrolled in HIIT also improve primary predictors of mortality and morbidity [12] as well as other predictors of relatively poor outcome [13] in chronic heart failure. Thus, the aim of this post hoc analysis was to evaluate the effects of a 6-month HIIT program on selected estimates of (1) functional capacity and health-related quality of life, (2) multiple blood biomarkers, (3) echocardiographic parameters, and (4) exercise performance, in patients stratified by the presence of AF with the intention of answering the following questions: (1) Does CRT provide similar benefits in patients in AF and SR?; and (2) Does HIIT optimize benefits in patients in AF and SR? 


\section{Methods}

\section{Study design}

This is a post hoc analysis of a single-center, randomized controlled trial aimed at determining the effects of a 6-month HIIT program initiated soon after CRT implantation (2-4 weeks) on selected noninvasive estimates of systolic function, exercise performance, the severity of symptoms and healthrelated quality of life (Clinicaltrials.gov: NCT02413151). Onehundred twenty-one patients with chronic heart failure and referred to CRT at Hospital Santa Marta were eligible for the trial. The inclusion criteria were: patients classified in NYHA functional class II-IV; receiving optimal medical therapy for chronic heart failure, with a stable condition for more than 1 month; reduced ejection fraction, and QRS duration $\geq 120 \mathrm{~ms}$. Exclusion criteria were: geographical long-distance address with difficulty/impossibility to attend the hospitalbased HIIT program, incapacitating orthopedic, neurologic or other limitations that unable the patient to exercise, not acceptance to participate in the study, inability to sign informed consent, previous treatment with an intravenous inotropic agent within the 30 days prior to implantation or unstable angina pectoris.

Patients were randomized and stratified (by gender, age and etiology) following CRT. The randomization code was developed with a computer random-number generator to select random permuted blocks. Fifty-eight patients were not randomized either because they declined to participate in the study or as a result of clinical changes during the CRT implantation. The remaining 63 patients with moderate to severe CHF (NYHA class II-IV who were under optimal medical therapy with QRS $>120 \mathrm{msec}$ ) were randomized to HIIT (HIIT, $n=34$ ) or to the usual care group (CON, $n=29$ ) who were not given specific advice on exercise training nor underwent supervised training. No change in cardiac medication occurred as part of the study protocol.

One patient from the HIIT group died during the study and 20 patients successfully completed the exercise protocol with attendance at $>80 \%$ of the 48 sessions and attended $100 \%$ of the evaluation visits. Three of the 29 control patients died during the study and 9 did not complete assessments. Therefore, 37 patients (HIIT, $n=20$; CON, $n=17$ ) completed the study. These 37 patients undergoing CRT with complete data on functional capacity and healthrelated quality of life, B-type natriuretic peptides (BNP), Creactive protein (CRP), creatinine and inflammatory cytokines, echocardiographic parameters, and exercise performance, were stratified by the presence of AF for the sole purpose of this rhythm post hoc analysis [HIIT (AF $=7$; SR $=11) ; \mathrm{CON}(\mathrm{AF}=9 ; \mathrm{SR}=10 ; 67.6 \pm 1.9$ years $)$. All patients in AF had persistent AF (3 months). All measurements were performed prior to CRT implantation and repeated 6 months following implantation. No change in cardiac medication occurred as part of the study protocol.

Optimal medical therapy for chronic heart failure was considered to include a diuretic, an angiotensin-converting enzyme inhibitor or an angiotensin receptor blocker and a beta-blocker, as recommended [14].

The trial was performed according to the Helsinki declaration, approved by the hospital medical ethics research committee. Written informed consent was obtained from all patients.

\section{Cardiopulmonary exercise testing}

A symptom-limited incremental cardiopulmonary exercise test (CPET), was performed on a treadmill (Bruce protocol) with breath-by-breath gas exchange measurements (InnocorR, Innovision, Cardiosolutions) and online realtime calculation of $\mathrm{VO}_{2}, \mathrm{CO}_{2}$ production and respiratory exchange ratio (RER). Subjects were tested at least 2 hours post-prandial and with their regular medication.

A 12-lead ECG was recorded continuously, and blood pressure was measured by auscultation using an aneroid sphygmomanometer (at rest, at the end of each stage and every min after peak effort).

Subjects were encouraged to exercise until exhaustion, as defined by the inability to keep up with the treadmill speed, by leg fatigue or dyspnea, and RER values $>1.1$ ) unless clinical criteria for earlier test termination were observed. Peak $\mathrm{VO}_{2}$ was considered the highest attained $\mathrm{VO}_{2}$ during the final $30 \mathrm{sec}$ of exercise (10 s average).

\section{Echocardiography parameters}

A complete transthoracic echocardiogram was performed in all patients using a Vivid E9 scanner (GE Healthcare) with a $3 \mathrm{MHz}$ probe, at baseline and at 6-month follow-up $[15,16]$. M-mode, two-dimensional, color, pulsed, continuous wave and tissue Doppler data were obtained from parasternal and apical views, and the standard echocardiographic parameters were calculated. Acquired cine-loop images with at least 3 cardiac cycles were analyzed offline (EchoPAC software, GE Healthcare) for additional measures. Left ventricular ejection fraction (LVEF), and systolic (LVESV) and diastolic (LVEDV) volumes were determined from apical 4- and 2-chamber views using Simpson's biplane method [17]. The mean of 3 measurements was considered for analysis. Endocardial borders were manually traced, and the left ventricular papillary muscles were included in volume acquisition.

\section{Blood biomarkers analysis}

Blood samples were collected through peripheral venous catheterization into blood collection tubes with anticoagulant (plasma) or without (serum). All laboratory tests were performed after at least $6 \mathrm{~h}$ fasting and at rest in a supine position in the same laboratory using hospital protocol, and assays were determined according to the manufacturer's recommendations. Concentrations of TNF- $\alpha$, IL- 6 were determined using commercially available ELISA kits (R\&D Systems, USA), BNP was analyzed from plasma on a Spinchron DLX 800 (Beckman Coulter) centrifuge using a 
two-step chemiluminescence assay, and CRP was determined with a nephelometer (Siemens BN ProSpecT). Serum creatinine levels were measured using the Jaffe method (Roche Diagnostics GmbH).

\section{Health-related quality of life}

Patient health-related quality of life was assessed using a validated Portuguese version of the HeartQoL, a 14-item self-administered questionnaire enabling respondents to recall how much their heart problem bothered them during the past four weeks, which has been validated in patients with chronic heart failure [18]. The HeartQoL is scored on a four-point Likert scale (0-3) with higher scores representing better health-related quality of life. A global, physical and emotional HeartQoL score can be calculated [18].

\section{Exercise training}

The HIIT was initiated soon after CRT implantation (2-4 weeks). Sessions were supervised, hospital-based, ECG monitored and implemented twice a week, each for $60 \mathrm{~min}$, on non-consecutive days for 6 months (48 sessions). During the first month, each interval training and the active pause were increased by $30 \mathrm{sec}$ on a weekly basis, $4 \mathrm{~min}$ of work with $3 \mathrm{~min}$ of active rest were accomplished. Every session included a 10-min warm-up and a 5-7 min cool-down. Starting from the second month, the HIIT consisted of 4 interval training periods (high intensity: $90-95 \%$ of maximal heart rate if below the device threshold, and if not, 90-95\% of the device threshold was used) with 3 lower-intensity active periods (moderate intensity: $60-70 \%$ of maximal heart rate if below the device threshold) between interval training periods [19]. The CON group was given no specific advice on exercise training and underwent no supervised training.

\section{Statistical analysis}

All data are reported as mean \pm standard error of the mean and statistical significance was established a priori as $p<.05$. Normality of distribution for variables was assessed qualitatively using histograms and Q-Q plots as well as quantitatively using the Shapiro-Wilk test, kurtosis and skewness summary statistics. When distribution was found to be skewed, log transformations were performed to conform to normality. Descriptive characteristics were compared using ANOVA for continuous variables and Qui-squared tests for categorical data.

Since this post hoc analysis was intended as an efficacy analysis, we focused on a per-protocol analyses, which included the patients in CRT that successfully completed the exercise protocol with attendance rates superior to $80 \%$. Differences between groups (HIIT AF, HIIT SR, CON AF and CON SR) at baseline and postintervention, changes over time within each group and any interaction effect were assessed by multiple mixed designed two-way repeated measures analysis of variance (ANOVA).
The statistical analyses were computed and analyzed using the SPSS Statistics 22.0 (SPSS Inc., Chicago, IL, USA).

\section{Results}

Baseline characteristics of the Patients undergoing CRT by heart rhythm control are displayed in Table 1.

The effects of 6 months of HIIT following CRT on Functional Capacity and health-related quality of life, serum inflammatory response, echocardiographic parameters and exercise performance, in AF and SR patients are presented in Table 2 by rhythm groups.

\section{Does CRT provide similar benefits in patients in AF and $S R$ ? [CON AF x CON SR]}

No differences were found in NYHA class and health-related QOL between patients in AF and SR at baseline or postintervention $(p>.05)$. Health-related QOL (82.8-101.1\%; $p<.001$; $\left.\eta^{2}=0.570\right)$ improved over time in both groups. However, a significant interaction effect was found in NYHA class (23.8-46.0\% $\left.p<.001 ; \eta^{2}=0.743 ; p=.034\right)$, as improvements were greater in patients in SR.

TNF- $\alpha$, BNP, CRP and Creatinine did not differ between patients in $\mathrm{AF}$ and $\mathrm{SR}$ at baseline or postintervention but serum concentrations of IL-6 were superior in AF compared to $\operatorname{SR}(p=.014)$. Still, no significant improvements were observed in serum BNP and inflammatory markers with device implantation in either group $(p>.05)$.

No differences were found in LVEF, LV Mass, LVEDV or LVESV between patients in AF and SR at baseline or postintervention $(p>.05)$. Increases in LVEF (31.1-58.8\%; $\left.p=.002 ; \eta^{2}=0.432\right)$ were observed with device implantation. LVESV, LVEDV and LV Mass did not change significantly with device implantation.

Peak HR, RER and peak $\mathrm{VO}_{2}$ did not differ between patients in $\mathrm{AF}$ and $\mathrm{SR}$ at baseline or postintervention and did no change over time with device implantation in any group $(p>.05)$. CPET duration was higher in patients in SR at baseline $(p=.018)$ but not at postintervention $(p=.232)$. Still, no significant changes were observed over time $(p=.433)$.

Table 1. Baseline characteristics of the patients undergoing CRT, by heart rhythm control.

\begin{tabular}{|c|c|c|c|}
\hline & Unit & Atrial Fibrillation & Sinus Rhythm \\
\hline Subjects & $n$ & 16 & 21 \\
\hline Age & Years & $69.4 \pm 1.8$ & $66.2 \pm 3.18$ \\
\hline Males & $\%$ & 78.5 & 75.0 \\
\hline Etiology & $\%$ & & \\
\hline Ischaemic & & 47.4 & 29.2 \\
\hline Dilated Cardiomyopathy & & 52.6 & 70.8 \\
\hline Medication & $\%$ & & \\
\hline Anticoagulants & & 86.7 & 61.9 \\
\hline$\beta$-Blockers & & 92.3 & 85.7 \\
\hline ACE Inhibitors & & 100 & 90.5 \\
\hline Diuretics & & 93.3 & 85.7 \\
\hline Body Mass Index & $\mathrm{Kg} / \mathrm{m}^{2}$ & $28.2 \pm 1.2$ & $26.7 \pm 1.0$ \\
\hline Heart rate & bpm & $77 \pm 4$ & $79 \pm 4$ \\
\hline Systolic Blood Pressure & $\mathrm{mmHg}$ & $116.8 \pm 5.0$ & $115.3 \pm 3.8$ \\
\hline Diastolic Blood Pressure & $\mathrm{mmHg}$ & $64.0 \pm 2.8$ & $62.7 \pm 2.1$ \\
\hline
\end{tabular}

Values are means $\pm S E$. 
Table 2. Effects of 6 months of HIIT following CRT by rhythm control.

\begin{tabular}{|c|c|c|c|c|c|}
\hline & \multirow[b]{2}{*}{ Unit } & \multicolumn{2}{|c|}{ Atrial Fibrillation } & \multicolumn{2}{|c|}{ Sinus Rhythm } \\
\hline & & HIIT & Control & HIIT & Control \\
\hline \multicolumn{6}{|l|}{ NYHA } \\
\hline Baseline & & $3.0 \pm 0.6$ & $2.6 \pm 0.4$ & $2.8 \pm 0.1$ & $2.9 \pm 0.1$ \\
\hline Post-Intervention & & $1.2 \pm 0.5^{+\Delta}$ & $2.0 \pm 0.8^{*+}$ & $1.4 \pm 0.2^{+\Delta}$ & $1.6 \pm 0.2^{*+}$ \\
\hline \multicolumn{6}{|l|}{ Quality of Life } \\
\hline Baseline & & $0.9 \pm 0.2$ & $0.8 \pm 0.2$ & $0.8 \pm 0.2$ & $1.0 \pm 0.2$ \\
\hline Post-Intervention & & $1.8 \pm 0.3^{+\Delta}$ & $1.7 \pm 0.3^{*+}$ & $1.9 \pm 0.1^{+\Delta}$ & $1.8 \pm 0.2^{*+}$ \\
\hline TNF- $\alpha$ & $\mathrm{pg} / \mathrm{mL}$ & & & & \\
\hline Baseline & & $2.4 \pm 0.6$ & $2.3 \pm 0.5$ & $3.0 \pm 0.5$ & $2.4 \pm 0.5$ \\
\hline Post-Intervention & & $2.1 \pm 0.6^{\Delta}$ & $2.3 \pm 0.5$ & $1.7 \pm 0.5^{\Delta}$ & $2.2 \pm 0.5$ \\
\hline BNP & $\mathrm{pg} / \mathrm{mL}$ & & & & \\
\hline Baseline & & $583.7 \pm 308.9$ & $935.8 \pm 338.4$ & $378.1 \pm 99.1$ & $404.9 \pm 99.1$ \\
\hline Post-Intervention & & $499.5 \pm 320.2^{\Delta}$ & $840.4 \pm 350.8$ & $320.1 \pm 79.4^{+\Delta}$ & $264.9 \pm 79.4^{+}$ \\
\hline IL-6 & $\mathrm{pg} / \mathrm{mL}$ & & & & \\
\hline Baseline & & $11.28 \pm 3.74$ & $10.66 \pm 3.34$ & $6.43 \pm 1.35$ & $3.45 \pm 1.11$ \\
\hline Post-Intervention & & $11.85 \pm 3.67$ & $6.16 \pm 3.28$ & $2.85 \pm 1.39$ & $4.61 \pm 1.13$ \\
\hline Creatinine & $\mathrm{mg} / \mathrm{L}$ & & & & \\
\hline Baseline & & $1.32 \pm 0.17$ & $1.31 \pm 0.16$ & $1.07 \pm 0.14$ & $0.96 \pm 0.15$ \\
\hline Post-Intervention & & $1.16 \pm 0.16$ & $1.35 \pm 0.15$ & $1.11 \pm 0.18$ & $1.09 \pm 0.20$ \\
\hline CRP & $\mathrm{mg} / \mathrm{L}$ & & & & \\
\hline Baseline & & $4.10 \pm 1.90$ & $3.86 \pm 1.77$ & $3.93 \pm 1.33$ & $3.14 \pm 1.45$ \\
\hline Post-Intervention & & $1.81 \pm 0.58$ & $1.98 \pm 0.55$ & $1.77 \pm 2.03$ & $6.21 \pm 2.22$ \\
\hline LVEF & $\%$ & & & & \\
\hline Baseline & & $27.1 \pm 3.0$ & $27.6 \pm 2.6$ & $26.7 \pm 2.2$ & $24.1 \pm 2.4$ \\
\hline Post-Intervention & & $31.9 \pm 4.7^{+}$ & $36.1 \pm 4.1^{*+}$ & $40.0 \pm 2.9^{+\Delta}$ & $36.5 \pm 3.2^{*+\Delta}$ \\
\hline LV Mass & $g$ & & & & \\
\hline Baseline & & $286.8 \pm 50.7$ & $401.8 \pm 50.7$ & $376.8 \pm 30.9$ & $335.4 \pm 36.9$ \\
\hline Post-Intervention & & $285.6 \pm 51.3$ & $356.8 \pm 51.3$ & $278.2 \pm 29.6^{+\Delta}$ & $303.1 \pm 35.4^{+\Delta}$ \\
\hline LV EDV & $\mathrm{ml}$ & & & & \\
\hline Baseline & & $251.8 \pm 48.0$ & $242.0 \pm 37.9$ & $169.3 \pm 32.7$ & $241.8 \pm 32.7$ \\
\hline Post-Intervention & & $200.4 \pm 44.8$ & $236.4 \pm 35.4$ & $191.9 \pm 32.7$ & $229.5 \pm 32.7$ \\
\hline LV ESV & $\mathrm{ml}$ & & & & \\
\hline Baseline & & $180.5 \pm 37.6$ & $180.0 \pm 32.6$ & $114.7 \pm 23.7$ & $182.0 \pm 23.7$ \\
\hline Post-Intervention & & $152.7 \pm 37.9$ & $158.1 \pm 32.9$ & $136.7 \pm 22.6$ & $147.3 \pm 22.6$ \\
\hline Peak HR & bpm & & & & \\
\hline Baseline & & $114 \pm 10$ & $139 \pm 11$ & $125 \pm 7$ & $128 \pm 7$ \\
\hline Post-Intervention & & $107 \pm 10$ & $118 \pm 12$ & $125 \pm 5$ & $129 \pm 5$ \\
\hline Peak $\mathrm{VO}_{2}$ & $\mathrm{ml} / \mathrm{kg} / \mathrm{min}$ & & & & \\
\hline Baseline & & $12.6 \pm 1.7$ & $12.1 \pm 1.7$ & $15.3 \pm 2.0$ & $18.7 \pm 2.1$ \\
\hline Post-Intervention & & $15.0 \pm 2.3^{+}$ & $15.6 \pm 2.3^{+}$ & $17.5 \pm 1.3$ & $18.1 \pm 1.3$ \\
\hline CPET Duration & seg & & & & \\
\hline Baseline & & $331 \pm 91$ & $258 \pm 99$ & $418 \pm 67$ & $614 \pm 75$ \\
\hline Post-Intervention & & $544 \pm 92^{\Delta}$ & $403 \pm 101$ & $666 \pm 54^{+\Delta}$ & $555 \pm 61$ \\
\hline RER & unitless & & & & \\
\hline Baseline & & $1.10 \pm 0.07$ & $0.99 \pm 0.07$ & $0.98 \pm 0.04$ & $0.99 \pm 0.04$ \\
\hline Post-Intervention & & $1.09 \pm 0.04$ & $1.00 \pm 0.04$ & $1.03 \pm 0.02$ & $0.97 \pm 0.03$ \\
\hline
\end{tabular}

Symbols indicate postintervention values are significantly different from baseline values $(p<.05)$ in: ${ }^{+}$HIIT vs Control in patients in SR or AF; ${ }^{\Delta}$ HIIT SR vs HIIT $\mathrm{AF} ; *$ Control SR vs Control AF. Values are means $\pm \mathrm{SEM}$.

HIIT: High Intensity Interval Training Group; NYHA: New York Heart Association Functional Class; TNF- $\alpha$ : Tumor necrosis factor; BNP: Brain natriuretic peptide; IL-6: Interleukin-6; CPR: C-reactive protein; LVEF: Left Ventricular Ejection Fraction; LV Mass: Left Ventricular Mass; LV EDV: Left Ventricular EndDiastolic Volume; LV ESV: Left Ventricular End-Systolic Volume; Peak HR: Heart rate at peak effort during cardiopulmonary exercise testing; Peak VO ${ }_{2}$ : Oxygen consumption at peak effort during cardiopulmonary exercise testing; CPET duration: Cardiopulmonary exercise testing duration; RER: Respiratory Exchange Ratio.

\section{Does HIIT provide benefits in patients in SR? [CON SR $\times$ HIIT SR]}

No differences were found in NYHA class and health-related QOL between intervention and control groups of patients in SR at baseline or postintervention $(p>.05)$. NYHA class (46.1-49.8\%; $\left.p<.001 ; \eta^{2}=0.853\right)$ and health-related QOL (86.1-145.6\%; $\left.p<.001 ; \eta^{2}=0.698\right)$ improved in both groups.

In patients in SR, TNF- $\alpha$, BNP, IL-6, CRP and Creatinine did not differ significantly between groups at baseline or postintervention. Decreases in TNF- $\alpha\left(8.5-42.9 \% ; p=.028 ; \eta^{2}=\right.$ $0.252)$ and $\operatorname{BNP}\left(15.3-34.6 \% ; p=.032 ; \eta^{2}=0.232\right)$ were observed in both groups, whereas no significant changes were observed in IL-6 and Creatinine.
No significant differences were found in LVEF, LV Mass, LVEDV or LVESV between HIIT and CON groups at baseline or postintervention. Increases in LVEF (50.0-51.5\%; $\left.p<.001 ; \eta^{2}=0.532\right)$ and decreases in LV Mass (9.6-26.2\%; $\left.p=.003 ; \eta^{2}=0.456\right)$ were observed in both groups (Figure 1(a,b)).

Peak HR, CPET duration, peak $\mathrm{VO}_{2}$ and RER did not differ significantly between at baseline or postintervention. Significant increases were observed in CPET duration (8.8-59.4\%; $\left.p=.014 ; \eta^{2}=0.325\right)$. However, an interaction effect was found ( $\left.p=.002 ; \eta^{2}=0.473\right)$, showing that CPET duration only increased in patients in SR enrolled in HIIT $(p<.001)$ (Figure 2(a,b)). 
(a) HIIT

Control

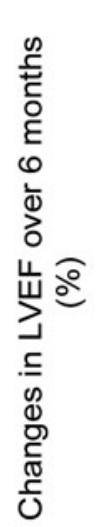

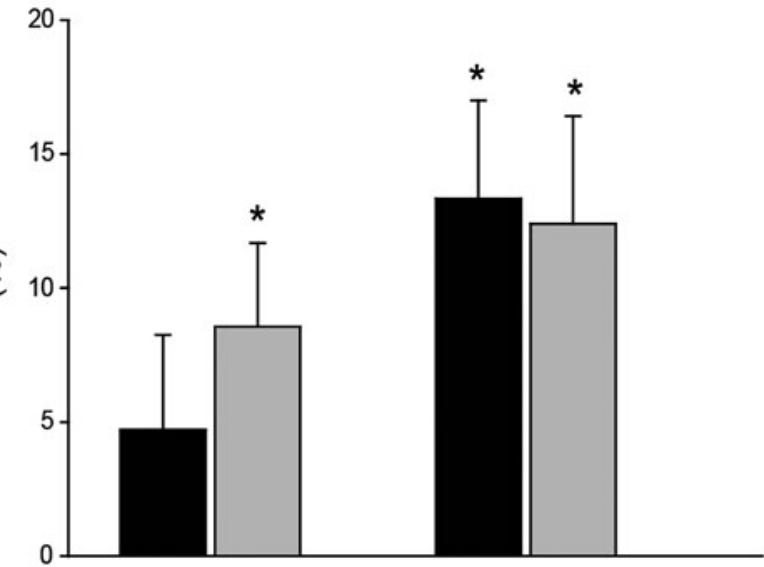

(b)

Atrial Fibrillation

Sinus Rhythm

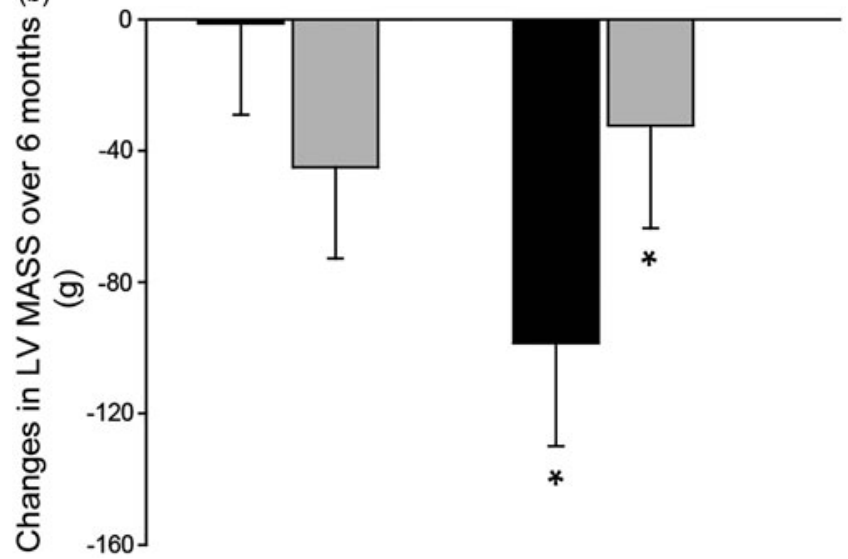

Figure 1. Effects of 6 months of HIIT following CRT (differences) in (a) LVEF and (b) LV Mass by rhythm control. * indicates post-intervention value is significantly different from baseline $(p<.05)$; Values are means \pm SEM. LVEF: Left Ventricular Ejection Fraction; LV Mass: Left Ventricular Mass.

\section{Does HIIT provide benefits in patients in AF? [CON AF $x$ HIIT AF]}

Patients in AF enrolled in HIIT had higher NYHA class at postintervention compared to those in $\operatorname{CON}(p=.032)$, but not at baseline $(p=.245)$. HRQOL did not differ between groups at any time point and improved equally in patients in both groups (101.1-106.3\%; $\left.p=.002 ; \eta^{2}=0.555\right)$. However, an interaction effect was observed in NYHA class in patients in $\mathrm{AF}$, so that improvements in functional capacity class were higher in those enrolled in HIIT $(23.9-61.0 \%$; $p=.004$; $\left.\eta^{2}=0.515\right)$.

TNF- $\alpha$, BNP, IL-6, CRP and Creatinine did not differ significantly among patients in AF at baseline or postintervention and did not improve significantly with time.

No significant differences were found in LVEF, LV Mass, LVEDV or LVESV among patients in AF at baseline or postintervention. Increases in LVEF $(17.4-31.1 \% ; p=.011$; $\eta^{2}=0.381$ ) were observed in both groups (Figure $1(\mathrm{a}, \mathrm{b})$ ). LVESV, LVEDV and LV Mass did not change significantly over time.

Peak HR, CPET duration, peak $\mathrm{VO}_{2}$ and RER did not differ significantly among patients in $\mathrm{AF}$ at baseline or postintervention. Significant increases in peak $\mathrm{VO}_{2}$ were observed (a) $\square$ HIIT $\square$ Control
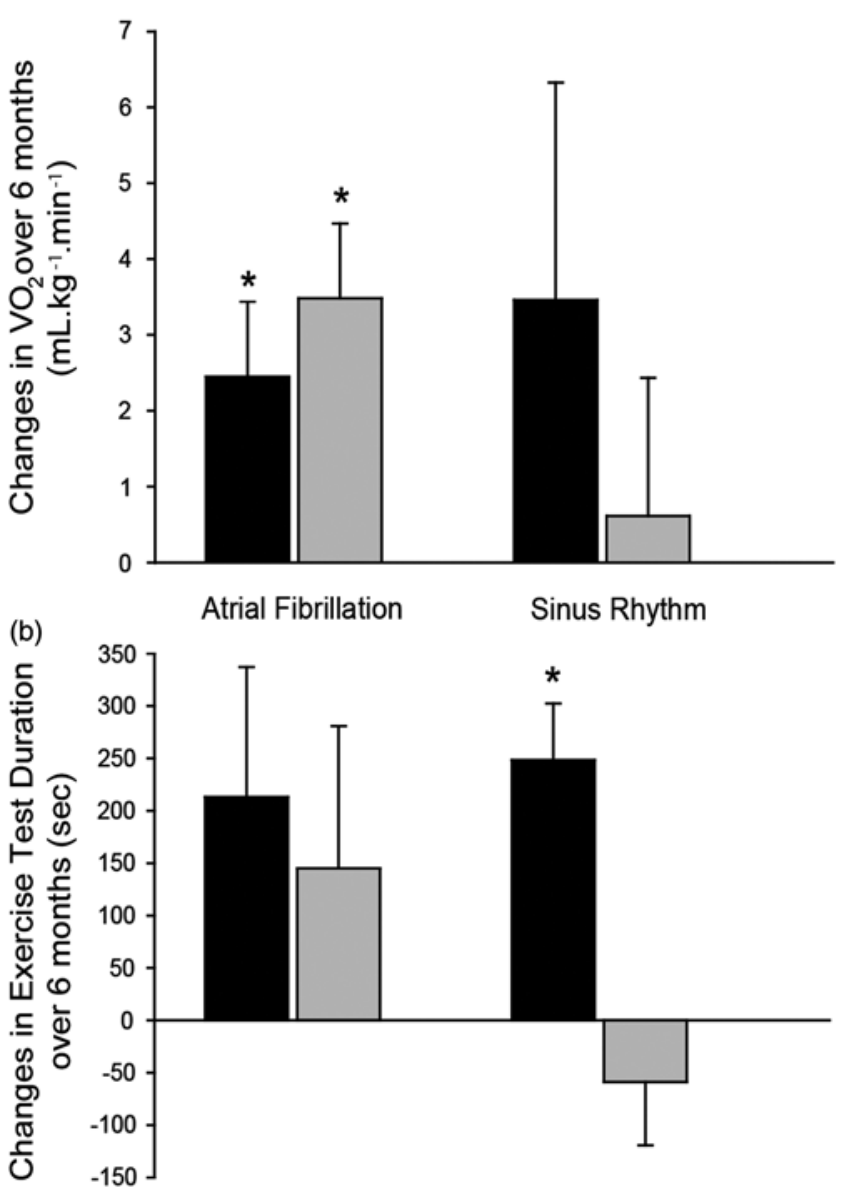

Figure 2. Effects of 6 months of HIIT following CRT (differences) in (a) peak $\mathrm{VO}_{2}$ and (b) CPET duration, by rhythm control. * indicates post-intervention value is significantly different from baseline $(p<.05)$; Values are means \pm SEM. Peak $\mathrm{VO}_{2}$ : Oxygen consumption at peak effort during cardiopulmonary exercise testing; CPET duration: Cardiopulmonary exercise testing duration.

in both groups $\left(19.5-23.2 \% ; p=.002 ; \eta^{2}=0.644\right.$; Figure 2(a,b)). Peak HR, CPET duration and RER did not change significantly over time.

\section{Does HIIT provide similar benefits in patients in AF and SR? [HIIT AF $\times$ HIIT SR]}

Improvements in NYHA class $\left(50.0-61.1 \% ; p<.001 ; \eta^{2}=\right.$ $0.883)$ and health-related QOL $\left(106.3-145.6 \% ; p<.001 ; \eta^{2}\right.$ $=0.697)$ were observed in both exercising groups.

TNF- $\alpha$, BNP, IL-6 CRP and Creatinine did not differ significantly between patients in AF and SR enrolled in HIIT at baseline or postintervention. HIIT decreased TNF- $\alpha$ $\left(11.6-42.9 \% ; p=.02 ; \eta^{2}=0.400\right)$ and BNP (14.4-15.3\%; $\left.p=.03 ; \eta^{2}=0.294\right)$ in both groups, whereas no significant changes were observed in IL-6, CRP and Creatinine.

No significant differences were found in LVEF, LV Mass, LVEDV or LVESV between patients in AF or SR enrolled in HIIT at baseline or postintervention. However, a significant interaction effect was observed $(p=.038)$, suggesting that LVEF increased $\left(50.0 \% ; p<.001 ; \eta^{2}=0.542\right)$ and LV Mass decreased $\left(26.2 \% ; p=.006 ; \eta^{2}=0.459\right)$ only 
in patients in SR. LVESV and LVEDV did not change significantly with HIIT.

Peak HR, CPET duration and RER did not differ significantly between patients in AF and SR enrolled in HIIT at baseline or postintervention. However, patients in $\mathrm{AF}$ had lower peak $\mathrm{VO}_{2}\left(2.50 \mathrm{~mL} \cdot \mathrm{kg}^{-1} \cdot \mathrm{min}^{-1}\right)$ compared to patients in SR at postintervention $(p=.041)$. Increases in CPET duration $\left(32.0-59.4 \% ; p=.007 ; \eta^{2}=0.0421\right)$ were observed in both groups.

\section{Discussion}

Despite the advancement in medicine, management of atrial fibrillation in patients with chronic heart failure remains a challenge to healthcare providers. This is reflected by the relatively higher rate of readmissions along with increased mortality and morbidity associated with AF [20]. In the present analysis, we evaluated the effects of a 6-month HIIT program on primary predictors of mortality and morbidity as well as other predictors of relatively poor outcome in patients in CRT stratified by the presence of AF. The main findings were that: 1) patients in $\mathrm{AF}$ benefitted to a lesser degree from CRT in functional status than patients in SR. However, HIIT induced superior improvements in patients in AF compared to CON; 2) decreases in TNF- $\alpha$, BNP, and left ventricular mass were only observed in patients in SR, whereas increases in peak oxygen uptake were only observed in patients in AF; 3) HIIT improved exercise capacity in patients in SR. We consider that these findings outline that both mainstay and adjunctive therapeutics may need to be managed differently in patients in AF and SR so as to meet the goals of the heart failure treatment. However, just as at present it is not clear whether $\mathrm{AF}$ is an independent risk factor or just a risk marker for increased mortality in patients with $\mathrm{CHF}$ and LV dysfunction, or that converting $\mathrm{AF}$ to $\mathrm{SR}$ and preventing the recurrence of $\mathrm{AF}$ is superior to a strategy aimed at preventing tachycardia during $\mathrm{AF}$ [21], we cannot claim that HIIT is an effective strategy to maximize benefits in patients in AF undergoing CRT.

It has been shown that CRT reduces serum levels of BNP [22] but our results suggest that heart rhythm may influence this finding as no changes were observed in patients in AF. However, interpreting BNP levels in patients with AF requires special consideration, unlike in patients in SR, as $\mathrm{BNP}$ in AF is the sum of the releases from the ventricle (reflecting left ventricular function) and the atrium (due to AF) [23]. Besides, not only chronic AF can affect BNP level, but also the effect of a paroxysmal AF attack that replaces SR may simply be reflected in the BNP levels [23]. Although CRT decreased BNP levels in patients in SR, HIIT did not add to the benefits of CRT. These findings are consistent with other studies with patients in AF or SR undergoing CRT $[24,25]$.

Tolosana et al. showed that the magnitude of functional improvement and reverse remodeling in patients in permanent AF undergoing CRT is similar to that experienced by patients in SR [26]. However, improvements in LVEF and LV Mass in our analysis were limited to patients in SR, and our data suggests that HIIT did not further improve these benefits. Several studies demonstrate that exercise training can improve physical fitness $[9,10,27,28]$, vascular function [29], quality of life [9], and, there is even evidence of reversing LV remodeling in clinically stable HF patients [30]. However, the evidence is conflicting as other studies reported no benefits with exercise training on ventricular hypertrophy, even with intense training [31,32]. The physiological mechanisms responsible for the remodeling effects of HIIT remains unknown. They may arise from favorable improvements, apparently exclusive to patients in SR, in coronary and peripheral vascular endothelial function [33], myocardial contractility [34], autonomic balance [35], or systolic and diastolic wall stress [36]. Subclinical target organ changes and cardiac remodeling could be the result of common pathophysiologic pathways [37]. Because large variation seen in some variables, possibly a function of low subject numbers, we adopted a conservative approach on our conclusions due to the risk of type I errors. Even though the effect sizes were large for most of our findings, studies with a larger sample size are needed to confirm these findings.

Patients undergoing CRT demonstrate both central and peripheral decompensation due to a prolonged inability to exercise. Therefore, the improvements found in healthrelated quality of life and NYHA are important since these patients are highly symptomatic, in particular patients in AF who have reduced survival and more advanced symptoms of heart failure [38]. The association between NYHA functional classes and poorer outcomes in patients with heart failure and left ventricular systolic dysfunction is widely recognized [39]. Consequently, the added benefits provided by HIIT in patients in AF have likely implications in the risk of hospitalization [40] due to reduction in symptoms of fatigue and dyspnea.

Patwala et al. [9] showed that moderate to vigorous exercise training in addition to CRT was a promising adjunctive therapeutic leading to further improvements in peak $\mathrm{VO}_{2}$ by improving skeletal muscle performance. However, in the present analysis we showed smaller increases in CPET duration and peak $\mathrm{VO}_{2}$ than in that study, and that these benefits were not independent of the heart rhythm control of the patients. This is important as the study by Patwala et al. [9] had a smaller percentage of patients with AF than the present analysis (34\% vs $44 \%$ ). Only a limited number of studies on exercise training in patients with AF have measured oxygen consumption and found that peak $\mathrm{VO}_{2}$ and exercise capacity increased correspondingly [41,42]. Our findings show that peak $\mathrm{VO}_{2}$ increased in patients in $\mathrm{AF}$ regardless of the allocation group, suggesting that HIIT did not provide further benefits in patients in AF undergoing CRT. Future studies should examine whether this finding might reflect the relatively small number of patients in our analysis or, differences in frequency, intensity or duration of the training program applied. Still, increases in peak $\mathrm{VO}_{2}$ of $\sim 20 \%$ are noteworthy in these patients and likely associated with the reported improvements in health-related quality of life. In the absence of an increase in peak $\mathrm{VO}_{2}$ 
at post-intervention in patients in SR, it is possible that improvements in health-related quality of life in this group derived from improvements in walking performance and efficiency factors, contributing to positive effects on the economy of motion [43] with expectable benefits in the performance of daily activities.

\section{Limitations}

The single-center and post hoc analysis design, the low number of participants, and the multiple testes performed are limitations of the present study. However, several strategies have been used to handle these multiple comparisons: (1) the eta squared effect size were strong and reported throughout the manuscript; (2) the conclusions do not advocate for any treatment decision. Instead, the authors looked for scientific plausibility and support data from other studies which validate the present results; (3) the authors focused on global assessment variables whenever possible such as NYHA functional class, LVEF, LV Mass or peak VO2. Still, this post hoc analysis was on average underpowered to demonstrate significant group differences in (1) functional capacity and health-related quality of life $(1-\beta=0.21 \pm 0.07)$, (2) multiple blood biomarkers $(1-\beta=0.36 \pm 0.08)$, (3) echocardiographic parameters $(1-\beta=0.34 \pm 0.01)$, and (4) exercise performance $(1-\beta=0.40 \pm 0.01)$.

Determination of several other blood biomarkers at both time points would have strengthened the discussion of our analysis. Unfortunately, no such samples were available from all patients.

Some observations suggest that atrioventricular node ablation may impact the attainment of maximal benefit from CRT in patients in AF [44]. Furthermore, exercise may revert $\mathrm{AF}$ to $\mathrm{SR}$ in some individuals. The mechanism is unclear, but possibly involves changes in autonomic tone affecting the atrium [45]. We do not have follow-up information on patients that might have been subjected to atrioventricular node ablation after CRT implantation or might have reverted to SR. Further studies are needed in patients who have atrioventricular node ablation and those who do not.

The ideal dose of exercise training regarding intensity and duration is still not known in patients undergoing CRT, with even less information in those with AF. We recognize that this exercise training program was more intense and longer than previous studies trying to maximize CRT benefits $[9,10]$, thus our findings add information on the longterm effect of HIIT in patients in AF.

\section{Conclusion}

Patients in AF or SR undergoing CRT demonstrated distinct benefits from device implantation and from HIIT as an adjunctive therapeutic strategy. This suggests that both mainstay and adjunctive therapeutics may need to be managed differently in patients in AF and SR so as to meet the goals of the heart failure treatment.

\section{Acknowledgements}

The authors would like to acknowledge the contributions of the cardiopulmonary technicians and the medical interns in cardiology from Hospital Santa-Marta, and Mafalda Carinha from Faculdade de Motricidade Humana - Universidade de Lisboa.

\section{Disclosure statement}

The authors report no conflict of interest. The authors take responsibility for all aspects of the reliability and freedom from bias of the data presented and their discussed interpretation.

All authors have made substantial contributions to all the following: (1) the conception and design of the study, or acquisition of data, or analysis and interpretation of data, (2) drafting the article or revising it critically for important intellectual content, (3) final approval of the version to be submitted.

\section{Authors' contributions}

HSC, AA contributed to conception and design, critically revised the manuscript, and gave final approval. VS contributed to acquisition, analysis, critically revised the manuscript and gave final approval. XM contributed to acquisition, analysis, interpretation, drafted the manuscript and gave final approval. RP and BF contributed to interpretation, critically revised manuscript, and gave final approval. PSC, MMO and MMC contributed to acquisition, critically revised the manuscript, and gave final approval.

\section{Funding}

This research was supported by National funding from the Portuguese Foundation for Science and Technology [PTDC/DES/120249/2010]. This funding body had no role in study design; in the collection, analysis and interpretation of data; in the writing of the report; and in the decision to submit the article for publication.

\section{ORCID}

Xavier Melo (D) http://orcid.org/0000-0003-3934-3986

\section{References}

[1] Abraham WT. Cardiac resynchronization therapy for the management of chronic heart failure. Amer Heart Hosp J. 2003;1: 55-61.

[2] Cleland JGF, Daubert J, Erdmann E, et al. The effect of cardiac resynchronization on morbidity and mortality in heart failure. N Engl J Med. 2005;352:1539-1549.

[3] Healey JS, Hohnloser SH, Exner DV, et al. Cardiac resynchronization therapy in patients with permanent atrial fibrillation: results from the Resynchronization for Ambulatory Heart Failure Trial (RAFT). Circ Heart Fail. 2012;5:566-570.

[4] Wilton SB, Leung AA, Ghali WA, et al. Outcomes of cardiac resynchronization therapy in patients with versus those without atrial fibrillation: a systematic review and meta-analysis. Heart Rhythm. 2011;8:1088-1094.

[5] Mullens W, Grimm RA, Verga T, et al. Insights from a cardiac resynchronization optimization clinic as part of a heart failure disease management program. J Am Coll Cardiol. 2009;53: 765-773.

[6] Gasparini M, Leclercq C, Lunati M, et al. Cardiac resynchronization therapy in patients with atrial fibrillation: the CERTIFY study (Cardiac Resynchronization Therapy in Atrial Fibrillation 
Patients Multinational Registry). JACC Heart Fail. 2013;1: 500-507.

[7] Gianni C, Di Biase L, Mohanty S, et al. How to improve cardiac resynchronization therapy benefit in atrial fibrillation patients: pulmonary vein isolation (and beyond). Heart Fail Clin. 2017;13:199-208.

[8] Delnoy PP, Ottervanger JP, Luttikhuis HO, et al. Comparison of usefulness of cardiac resynchronization therapy in patients with atrial fibrillation and heart failure versus patients with sinus rhythm and heart failure. Am J Cardiol. 2007;99:1252-1257.

[9] Patwala AY, Woods PR, Sharp L, et al. Maximizing patient benefit from cardiac resynchronization therapy with the addition of structured exercise training: a randomized controlled study. J Am Coll Cardiol. 2009;53:2332-2339.

[10] Belardinelli R, Capestro F, Misiani A, et al. Moderate exercise training improves functional capacity, quality of life, and endothelium-dependent vasodilation in chronic heart failure patients with implantable cardioverter defibrillators and cardiac resynchronization therapy. Eur J Cardiovasc Prev Rehabil. 2006;13:818-825.

[11] Santa-Clara H, Abreu A, Melo X, et al. High intensity interval training in cardiac resynchronization therapy - a randomized control trial. Eur J Appl Physiol. 2019. doi:10.1007/s00421-01904165-y.

[12] Upadhya B, Haykowsky MJ, Eggebeen J, et al. Exercise intolerance in heart failure with preserved ejection fraction: more than a heart problem. J Geriatr Cardiol 2015;12:294-304.

[13] Yancy CW, Jessup M, Bozkurt B, et al. ACCF/AHA guideline for the management of heart failure: a report of the American College of Cardiology Foundation/American Heart Association Task Force on Practice Guidelines. J Am Coll Cardiol. 2013;62: e147-239.

[14] Ponikowski P, Voors AA, Anker SD, et al. ESC Guidelines for the diagnosis and treatment of acute and chronic heart failure: the Task Force for the diagnosis and treatment of acute and chronic heart failure of the European Society of Cardiology (ESC) Developed with the special contribution of the Heart Failure Association (HFA) of the ESC. Eur Heart J. 2016;37:2129-2200.

[15] Evangelista A, Flachskampf F, Lancellotti P, et al. European Association of Echocardiography recommendations for standardization of performance, digital storage and reporting of echocardiographic studies. Eur J Echocardiogr. 2008;9:438-448.

[16] Lang RM, Badano LP, Mor-Avi V, et al. Recommendations for cardiac chamber quantification by echocardiography in adults: an update from the American Society of Echocardiography and the European Association of Cardiovascular Imaging. Eur Heart J Cardiovasc Imaging. 2015;16:233-270.

[17] Schiller NB, Shah PM, Crawford M, et al. Recommendations for quantitation of the left ventricle by two-dimensional echocardiography. American Society of Echocardiography Committee on Standards, Subcommittee on Quantitation of Two-Dimensional Echocardiograms. J Am Soc Echocardiogr. 1989;2:358-367.

[18] Oldridge N, Höfer S, McGee H, et al. The HeartQoL: part II. Validation of a new core health-related quality of life questionnaire for patients with ischemic heart disease. Eur J Prev Cardiolog. 2014;21:98-106.

[19] Wisloff U, Stoylen A, Loennechen JP, et al. Superior cardiovascular effect of aerobic interval training versus moderate continuous training in heart failure patients: a randomized study. Circulation. 2007;115:3086-3094.

[20] Olsson LG, Swedberg K, Ducharme A, et al. Atrial fibrillation and risk of clinical events in chronic heart failure with and without left ventricular systolic dysfunction: results from the Candesartan in Heart failure-Assessment of Reduction in Mortality and morbidity (CHARM) program. J Am Coll Cardiol. 2006;47:1997-2004.

[21] Neuberger HR, Mewis C, van Veldhuisen DJ, et al. Management of atrial fibrillation in patients with heart failure. Eur Heart J. 2007;28:2568-2577.
[22] Lappegård KT, Bjørnstad H. Anti-inflammatory effect of cardiac resynchronization therapy. Pacing Clin Electrophysiol. 2006;29:753-758.

[23] Tsuchida K, Tanabe K. Influence of paroxysmal atrial fibrillation attack on brain natriuretic peptide secretion. J Cardiol. 2004;44:1-11.

[24] Osbak PS, Mourier M, Kjaer A, et al. A randomized study of the effects of exercise training on patients with atrial fibrillation. Am Heart J. 2011;162:1080-1087.

[25] Nilsson BB, Westheim A, Risberg MA, et al. No effect of group-based aerobic interval training on $\mathrm{N}$-terminal pro- $\mathrm{B}$ type natriuretic peptide levels in patients with chronic heart failure. Scand Cardiovasc J. 2010;44:223-229.

[26] Tolosana JM, Hernandez Madrid A, Brugada J, et al. Comparison of benefits and mortality in cardiac resynchronization therapy in patients with atrial fibrillation versus patients in sinus rhythm (Results of the Spanish Atrial Fibrillation and Resynchronization [SPARE] Study). Am J Cardiol. 2008;102: 444-449.

[27] Smolis-Bạk E, Dąbrowski R, Piotrowicz E, et al. Hospital-based and telemonitoring guided home-based training programs: effects on exercise tolerance and quality of life in patients with heart failure (NYHA class III) and cardiac resynchronization therapy. A randomized, prospective observation. Int J Cardiol. 2015;199:442-447.

[28] Conraads VM, Vanderheyden M, Paelinck B, et al. The effect of endurance training on exercise capacity following cardiac resynchronization therapy in chronic heart failure patients: a pilot trial. Eur J Cardiovasc Prev Rehabil. 2007;14:99-106.

[29] Hambrecht R, Hilbrich L, Erbs S, et al. Correction of endothelial dysfunction in chronic heart failure: additional effects of exercise training and oral L-arginine supplementation. J Am Coll Cardiol. 2000;35:706-713.

[30] Haykowsky MJ, Liang Y, Pechter D, et al. A meta-analysis of the effect of exercise training on left ventricular remodeling in heart failure patients: the benefit depends on the type of training performed. J Am Coll Cardiol. 2007;49:2329-2336.

[31] Benda NM, Seeger JP, Stevens GG, et al. Effects of high-intensity interval training versus continuous training on physical fitness, cardiovascular function and quality of life in heart failure patients. PLoS One. 2015;10:e0141256.

[32] Myers J, Wagner D, Schertler T, et al. Effects of exercise training on left ventricular volumes and function in patients with nonischemic cardiomyopathy: application of magnetic resonance myocardial tagging. Am Heart J. 2002;144: 719-725.

[33] Sabelis LW, Senden PJ, Fijnheer R, et al. Endothelial markers in chronic heart failure: training normalizes exercise-induced vWF release. Eur J Clin Invest. 2004;34:583-589.

[34] Belardinelli R, Georgiou D, Cianci G, et al. Effects of exercise training on left ventricular filling at rest and during exercise in patients with ischemic cardiomyopathy and severe left ventricular systolic dysfunction. Am Heart J. 1996;132: 61-70.

[35] Coats AJ, Adamopoulos S, Radaelli A, et al. Controlled trial of physical training in chronic heart failure. Exercise performance, hemodynamics, ventilation, and autonomic function. Circulation. 1992;85:2119-2131.

[36] Demopoulos L, Bijou R, Fergus I, et al. Exercise training in patients with severe congestive heart failure: enhancing peak aerobic capacity while minimizing the increase in ventricular wall stress. J Am Coll Cardiol. 1997;29:597-603.

[37] Chahal H, Backlund J-Y, Cleary PA, et al. Relation between carotid intima-media thickness and left ventricular mass in type 1 diabetes mellitus (from the Epidemiology of Diabetes Interventions and Complications [EDIC] Study). Am J Cardiol. 2012;110:1534-1540.

[38] Crijns HJ, Tjeerdsma G, de Kam PJ, et al. Prognostic value of the presence and development of atrial fibrillation in patients 
with advanced chronic heart failure. Eur Heart J. 2000;21: 1238-1245.

[39] Bouvy ML, Heerdink ER, Leufkens HG, et al. Predicting mortality in patients with heart failure: a pragmatic approach. Heart. 2003;89:605-609.

[40] Perez-Moreno AC, Jhund PS, Macdonald MR, et al. Fatigue as a predictor of outcome in patients with heart failure: analysis of CORONA (Controlled Rosuvastatin Multinational Trial in Heart Failure). JACC Heart Fail. 2014;2:187-197.

[41] Vanhees L, Schepers D, Defoor J, et al. Exercise performance and training in cardiac patients with atrial fibrillation. J Cardiopulm Rehabil. 2000;20:346-352.
[42] Malmo V, Nes BM, Amundsen BH, et al. Aerobic interval training reduces the burden of atrial fibrillation in the short term: a randomized trial. Circulation. 2016;133:466-473.

[43] Beneke R, Meyer K. Walking performance and economy in chronic heart failure patients pre and post exercise training. Eur J Appl Physiol Occup Physiol. 1997;75:246-251.

[44] Molhoek SG, Bax JJ, Bleeker GB, et al. Comparison of response to cardiac resynchronization therapy in patients with sinus rhythm versus chronic atrial fibrillation. Am J Cardiol. 2004;94:1506-1509.

[45] Gates P, Al-Daher S, Ridley D, et al. Could exercise be a new strategy to revert some patients with atrial fibrillation? Intern Med J. 2010;40:57-60. 